\title{
Gas and dust in the most distant quasars
}

\author{
R. Maiolino ${ }^{1}$ \\ ${ }^{1}$ INAF - Osservatorio Astrofisico di Arcetri, Largo E. Fermi 5, Firenze, Italy \\ email: maiolino@arcetri.astro.it
}

\begin{abstract}
We use low resolution, near-IR spectra of eight of the most distant quasars known, with redshifts in the range $4.9<\mathrm{z}<6.4$, to investigate the gas and dust properties in their circumnuclear regions. Half of these quasars are characterized by deep, broad and blueshifted absorption features associated with both high and low ionization species, i.e. they belong to the class of Broad Absorption Line (BAL) quasars, which are associated with powerful outflows of dense gas. The large fraction of BAL quasars, the depth and ionization state of the absorption features suggest that these most distant quasars are surrounded by a much larger amount of dense gas than lower redshift $(\mathrm{z}<4)$ quasars. We discuss the possible interpretation of this result in terms of extremely high accretion rates and the association with the early formation of quasars.

The spectral shape of all these very distant quasars, and in particular of the BAL quasars, is bluer than for quasars at $\mathrm{z}<4$, suggesting an evolution of the properties of the dust responsible for the quasar reddening. We show that the observed spectral shape can be explained by an extinction curve due to dust produced by SNe. This finding would address the problem of the dust production at $\mathrm{z}>6$, when the age of the universe was lower than the evolutionary timescales of AGB stars.

Finally, these spectra allow to investigate the $\mathrm{Fe} / \alpha$-element abundance ratio, which is a tracer of the star formation history, through the relative strengths of the FeII UV bump and the MgII doublet. We find indications for a large abundance of Fe even in the most distant quasars at $\mathrm{z} \sim 6$. This finding requires that a strong burst of star formation occurred in the hosts of these quasars already at $\mathrm{z}>9$.
\end{abstract}

\section{Introduction}

The exceptional luminosities of quasars allow us to study the properties of gas and dust in their circumnuclear region even in the highest redshift systems known. However, for the most distant quasars discovered so far $(\mathrm{z} \sim 6)$ several of the most interesting spectral features are shifted into the near-IR, where observations are more difficult because of higher background, atmospheric transmission and limited spectral coverage of most spectrometers.

The Near Infrared Camera Spectrometer (NICS) at the Telescopio Nazionale Galileo (TNG) offers a low resolution, high sensitivity spectroscopic mode which is optimal to investigate the spectral properties of quasars at very high redshift. This mode makes use of an Amici prims (Oliva 2003), which allows us to obtain low resolution $(\mathrm{R}=50$ $100)$ spectra of the full near-IR range from $0.8 \mu \mathrm{m}$ to $2.5 \mu \mathrm{m}$, with very high throughput $(\sim 90 \%)$.

Such an observing mode turned out to be excellent to investigate several spectral features of the most distant quasars, and in particular: broad emission and absorption lines, continuum spectral shape (including dust reddening), and very broad features such as the UV and optical Fe bumps.

Here we discuss the results obtained from NICS-Amici spectra of 23 quasars in the redshift range $3.0<\mathrm{z}<6.4$ (although we will focus mostly on a subsample of 8 quasars at 


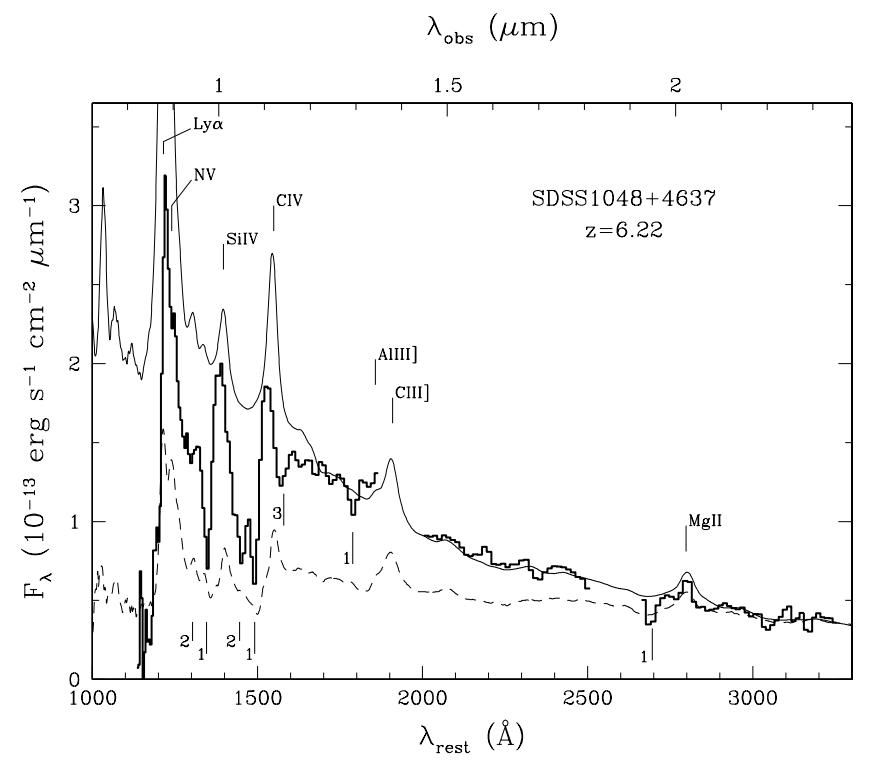

Figure 1. Spectrum of the most distant BAL quasars known. Two main absorption systems are identified with "1" and "2". The presence of AlIII and MgII absorption indicates that this is actually a LoBAL. The thin solid line is the (unreddened) non-BAL template with a slope $\alpha=-2.1$. The dashed line is the average spectrum of LoBAL quasars at $\mathrm{z}<4$. Further details are given in Maiolino et al. (2004a)

$\mathrm{z}>4.9$ ), and in particular the implications on the circumnuclear dust and gas properties and their relation with the star formation and host galaxy evolution. Most of these results are presented in Maiolino et al. (2003) and in Maiolino et al. (2004a,b)

\section{The large fraction of $B A L$ quasars at $\mathrm{z} \sim 6$}

Broad Absorption Line (BAL) quasars represent about $15 \%$ of the whole quasar population at "low" redshift ( $\mathrm{z}<4$, Reichard et al. 2003). These quasars are characterized by deep, broad and blueshifted absorption features associated with UV resonant lines of highly ionized species; in particular, $\operatorname{CIV}(1549 \AA)$ is generally the most prominent absorption feature. The commonly accepted scenario is that the broad absorption features are due to a strong wind of dense, ionized gas along our line of sight. Although orientation probably plays a role (the wind may be mostly equatorial), such strong outflows are also believed to be associated with high accretion rates (e.g. King \& Pounds 2003). A small fraction of BALs ( $\sim 2 \%$ of the whole quasar population) also show absorption associated with low ionization lines (AlIII and/or MgII); these are called Low ionization BALs (or simply LoBALs), and are probably characterized by larger columns of gas along our line of sight.

Eight of the quasars in our sample are at $z>4.9$; for these quasars CIV is redshifted in our spectral range and allows us to identify any CIV absorption. Our spectra show that four out of these eight quasars are characterized by broad, blueshifted CIV absorption, and are therefore BAL quasars (Maiolino et al. 2004a). Although the statistics is limited, this result is quite surprising when compared with the low occurrence (15\%) of BALs at lower redshifts $(z<4)$, and suggests that the fraction of BAL increases strongly at $z>4.9$. Fig. 1 shows the Amici spectrum of the most distant of these BAL quasars at $\mathrm{z}=6.22$, 
characterized by spectacular (double-) troughs of CIV and SiIV. Absorption of AlIII and $\mathrm{MgII}$ is also detected, indicating that this is a LoBAL. The sample contains an additional LoBAL at $\mathrm{z}=4.92$ (Maiolino et al. 2004a). Finding 2 LoBALs out of 8 quasars is an even more surprising result, since at $\mathrm{z}<4$ this fraction is about $2 \%$. An additional unexpected result is that most of these BAL quasars at $\mathrm{z}>4.9$ are characterized by extremely deep absorption troughs (indicative of large columns of gas). In particular for three of them $\mathrm{EW}\left(\mathrm{CIV}_{a b s}\right)>10 \AA$, which is quite rare at $\mathrm{z}<4$ (where only $15 \%$ of BAL have as deep CIV absorption).

Although the statistics is limited, the large fraction of BAL quasars, and specifically of LoBAL quasars, along with the deep troughs, strongly suggest that at z $5-6$ quasars are surrounded by larger amount of denser gas with respect to lower redshift quasars. Such strong outflows of dense gas are probably associated with the extreme accretion rates characterizing these primordial quasars. Strong quasars winds and high accretion rates are expected by the models of co-evolution of quasars and host galaxies at high redshift (Granato et al. 2004).

\section{Dust in the early universe}

Within the SDSS quasar survey Richards et al. (2003) found a number quasars at $\mathrm{z}<4$ with redder continua, a fraction which are well described in terms of reddening with an SMC-like extinction curve. BAL quasars are generally reddened by dust, probably associated with the outflowing gas, and in particular all LoBAL quasars appear reddened by dust (again with an SMC-like extinction curve).

At $\mathrm{z} \sim 5-6$ dust reddening appears to change significantly. Our near-IR spectra of these very distant quasars is that their spectral shape is generally bluer than lower redshift quasars. This is particularly interesting for the LoBAL quasars and in particular for the most distant of them in our sample (Fig.1), which is much bluer than any LoBAL quasar at $\mathrm{z}<4$ (the average LoBAL spectrum at $\mathrm{z}<4$ is shown with a dashed line). At least at $\lambda_{\text {rest }}>1700 \AA$ the spectral shape is consistent with the non-BAL template with slope $\alpha=-2.1\left(\mathrm{~F}_{\lambda} \propto \lambda^{\alpha}\right.$; thin solid line). This spectrum may suggest that this LoBAL at $\mathrm{z}=6.22$ is not significantly reddened by dust, at variance with all LoBAL qusars at $\mathrm{z}<4$.

Naively, this finding may indicate that at such high redshift the dust content in quasars is much lower than at $\mathrm{z}<4$. According to the standard scenario, dust is mostly formed in the envelopes of AGB stars, which require more than 1 Gyr to evolve. At $z=6.22$ the age of the universe is less than $900 \mathrm{Myr}$, therefore AGB stars had no time to produce large quantities of dust. Are we probing, for the first time, the dust-free Universe? This is certainly not the case, since $\mathrm{mm}$ and submm observations have revealed the emission from large masses of dust in these distant quasars (and in particular in the LoBAL at $\mathrm{z}=6.22$, Bertoldi et al. 2003, Priddey et al. 2003). The dust properties in these very distant object are therefore quite puzzling.

Some light is shed by the spectral shape at $\lambda_{\text {rest }}<1700 \AA$. In this spectral region the spectrum shows a bending (reddening) which was initially ascribed to deep absorption features. However the latter explanation is not satisfactory for various reasons. First, this would require that the SiIV absorption is deeper than CIV, which has never been observed in other BALs. Second, the spectrum bends already between $1700 \AA$ and CIV, where the CIV troughs does not play a role. Finally in a recent higher resolution spectrum (which will appear in Maiolino et al. 2004b) we clearly disentangle the continuum between the troughs, which appears to be redder than the extrapolation of the non-BAL unreddened quasar (thin line in Fig.1). Therefore, the spectrum is really reddened at $\lambda_{\text {rest }}<1700 \AA$. If this reddening is due to dust, then the associated extinction curve must be quite 
unusual: it must be relatively flat at $\lambda_{\text {rest }}>1700 \AA$ (not to redden the spectrum) and steeply rising at $\lambda_{\text {rest }}<1700 \AA$. This kind of extinction curve has never been observed so far.

Such a weird extinction curve must reflect some peculiar properties of the dust at $\mathrm{z} \sim 6$. As mentioned above, AGB stars cannot produce large quantities of dust at such high redshift. An alternative mechanism of dust production must be sought. Todini \& Ferrara (2001) demonstrated that the supernova ejecta offer a good environment of the formation of dust. This theoretical prediction has been supported by the recent detection of dust in some Galactic SN remnants (Dunne et al. 2003, Eales et al. 2003). Since the first core-collapse SNe occur on very short time scales ( 10 Myr) after the onset of a star formation episode, they can provide a fast and efficient source of dust in the early universe. We have derived the extinction curve expected from dust produced by SNe, and found it to be in excellent agreement with the one derived for the LoBAL quasar at $\mathrm{z}=6.22$ (this comparison will be shown in a forthcoming paper, Maiolino et al. 2004b). This finding indicates, for the first time, that at $\mathrm{z} \sim 6$ dust has been freshly produced by the first SNe occurring in the hosts of these distant quasars.

\section{Metallicity and star formation traced by the most distant quasars}

Quasar spectra are characterized by a prominent hump at 2200-3100A due to a blend of several hundreds FeII (and some FeIII) lines. Iron is potentially an excellent tracer of the star formation history, since it is mostly produced by type Ia SNe. For this reason, according to the standard chemical evolutionary scenarios, more than 1-1.5 Gyr is required to enrich the ISM with iron. Instead, $\alpha$-elements (e.g. Mg) are mostly produced by type II SNe, which evolve on much shorter time scales ( $10 \mathrm{Myr})$. Therefore, the ratio Fe/ $\alpha$ has been regarded as a "clock" of the star formation history (Hamann \& Ferland 1999). In particular, $\mathrm{Fe} / \alpha$ is expected to decrease at $\mathrm{z}>4$, i.e. when the age of the universe is approaching 1-1.5 Gyr.

The ratio of FeII/MgII $(\lambda 2798)$ is sensitive to the relative abundance Fe/ $\alpha$. Both species come from similar regions (they have similar ionization potentials). However, FeII is an important coolant of the low-ionization part of the BLR in quasars, and therefore the intensity of the bump is not expected to depend linearly from the Fe abundance. Corbin et al. (this conference) have calculated the expected trend of FeII/MgII as a function of $\mathrm{Fe} / \alpha$ abundance ratio, that indeed results to be not linear, but at least monotonic. Therefore the ratio FeII/MgII can be regarded as tracer of relative variations of the $\mathrm{Fe} / \alpha$ abundance, under the hypothesis that the physical conditions of the BLR do not change strongly (on average) with redshift (see Dietrich et al. 2003 for a more detailed discussion). More recently, Verner et al. (2004) and Verner \& Peterson (2004) have argued that the FeII/MgII may actually depend much more on the physical conditions of the BLR than on metallicity effects. In particular, they can explain extreme FeII/MgII ratios even with solar metallicities, but assuming very high densities, exceeding $10^{-11} \mathrm{~cm}^{-3}$. Such high densities may apply to the highly ionized part of the BLR (emitting Ly $\alpha$, CIV, etc...), but most likely does not apply to the lower ionization region of the BLR, since the presence of the strong semi-forbidden line CIII $](\lambda 1909)$ requires the low-ionization part of the BLR to have densities below $10^{-10} \mathrm{~cm}^{-3}$.

The measurement of the FeII UV bump when shifted into the near IR (i.e. $z>3$ ) is often difficult. Indeed this feature is very broad, and the limited spectral coverage of several near-IR spectrometers does not allow to properly determine the underlying continuum by interpolating from the contiguous spectral regions. Instead, the huge spectral coverage of NICS-Amici allows an excellent determination of the continuum underlying the $\mathrm{Fe}$ 


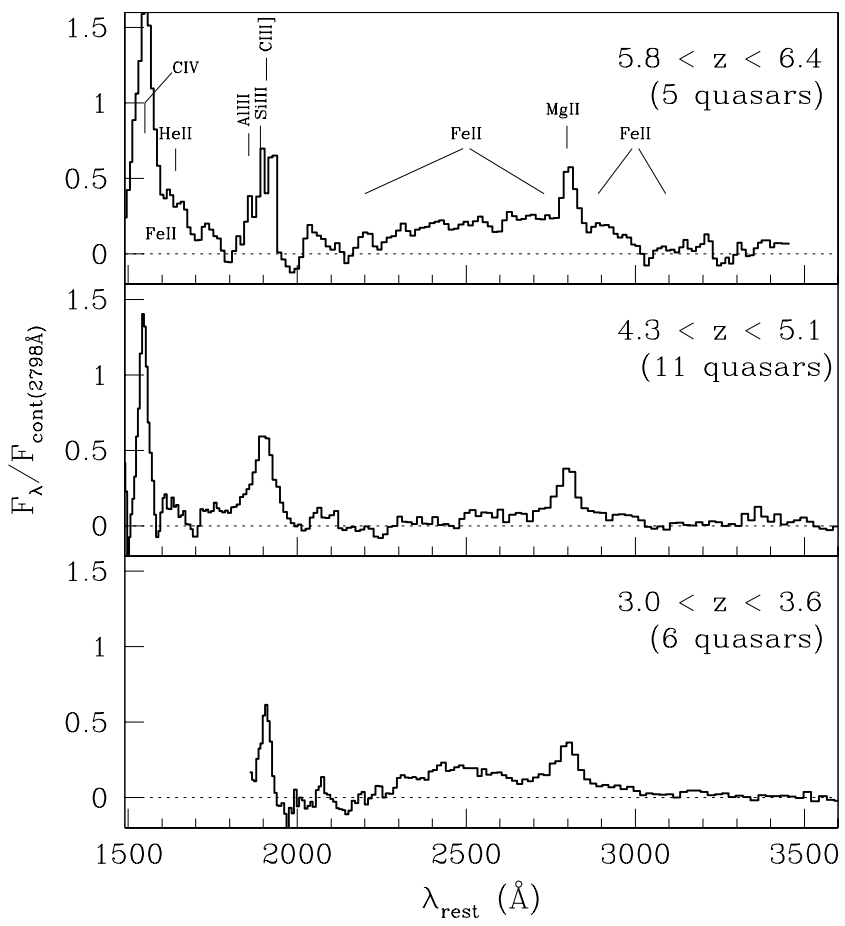

Figure 2. Continuum subtracted spectra of the quasars in our sample averaged within three main redshift bins. Note the strong iron emission in quasars at $\mathrm{z} \sim 6$. Further details are given in Maiolino et al. (2003)

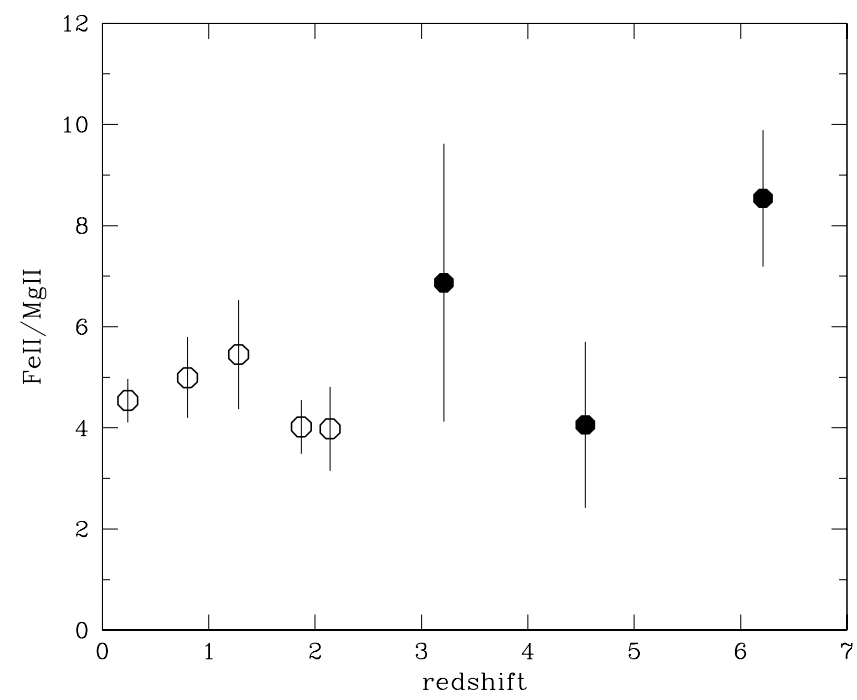

Figure 3. Filled symbols show the FeII/MgII ratio of the quasars in our sample averaged in redshift bins. Hollow symbols show the FeII/MgII ratio obtained by Dietrich et al. (2003) from optical composite spectra of quasars at lower redshift. 
bump, and an accurate determination of its intensity. Fig.2 shows continuum-subtracted spectra of the quasars in our sample, which have been averaged in three main redshift bins (Maiolino et al. 2003). The most important result is the strong iron emission in the group of quasars at $\mathrm{z} \sim 6$ (top panel), which indicates that large quantities of iron has already been produced at such high redshift. Fig.3 shows the FeII/MgII ratio as a function of redshift obtained by combining our results at $\mathrm{z}>3$ with the lower redshift data from Dietrich et al. (2003). The FeII/MgII does not show any decline beyond $\mathrm{z}>4$ up to $\mathrm{z} \sim 6$, which was expected by the classical chemical evolutionary models.

Matteucci \& Recchi (2001) have shown that high iron enrichment can actually occur on times scales shorter than $1 \mathrm{Gyr}$, provided that star formation is highly efficient and proceeds very rapidly. In particular, a simple model of early of formation of ellipticals can produce a peak of SNIa (therefore of iron enrichment) already at 0.3 Gyr after the onset of star formation. Within the latter scenario, the high iron enrichment observed in quasars at $\mathrm{z} \sim 6$ can be explained with a major episode of star formation, in the hosts of these quasars, which occurred at $z>9$. The latter is consistent with the redshift of the re-ionization $(z \approx 20 \pm 10)$ inferred from the recent WMAP results (Bennet et al. 2003).

\section{Acknowledgements}

The results presented in this paper were obtained in collaboration with several other astronomers, and in particular: S. Bianchi, A. Ferrara, F. Ghinassi, Y. Juarez, F. Mannucci, R. Mujica, N. Nagar, E. Oliva, M. Pedani, M. Roca Sogorb, R. Schneider. This work was partially supported by the Italian Institute for Astrophysics (INAF) and by the Italian Ministry of Research (MIUR).

\section{References}

Bertoldi, F., Carilli, C. L., Cox, P., Strauss, M. A., Beelen, A., Omont, A., \& Zylka, R. 2003, A\&A, 406, L55

Bennett, C. L., et al. 2003, ApJ, 583, 1

Dietrich, M., Hamann, F., Appenzeller, I., \& Vestergaard, M. 2003, ApJ, 596, 817

Dunne, L., Eales, S. Ivison, R., Morgan, H., \& Edmunds, M. 2003, Nature, 424, 283

Eales, S., Bertoldi, F., Ivison, R., Carilli, C., Dunne, L., \& Owen, F. 2003, MNRAS, 344, 169

Granato, G. L., De Zotti, G., Silva, L., Bressan, A., \& Danese, L. 2004, ApJ, 600, 580

Hamann, F., \& Ferland, G. 1999, ARA\&A, 37, 487

King, A. R., \& Pounds, K. A. 2003, MNRAS, 345, 657

Maiolino, R., Juarez, Y., Mujica, R., Nagar, N. M., \& Oliva, E. 2003, ApJ, 596, L155

Maiolino, R., Oliva, E., Ghinassi, F., Pedani M., Mannucci, F., Mujica, R., \& Juarez, Y. 2004a, $\mathrm{A} \& \mathrm{~A}$, in press (astro-ph/0312402)

Maiolino, R., et al. 2004a, A\&A, submitted

Matteucci, F., \& Recchi, S. 2001, ApJ, 401, 519

Oliva, E. 2003, Mem.S.A.It, 74, 118

Priddey, R. S. Isaak., K. G., McMahon, R. G., Robson, E. I., \& Pearson, C. P. 2003, MNRAS, 344, L74

Reichard, T. A., et al. 2003, AJ, 125, 1711

Richards, G., et al. 2003, AJ, 126, 1131

Todini, P., \& Ferrara, A. 2001, MNRAS, 325, 726

Verner, E., Bruhweiler, F., Verner, D., Johansson, S., Kallman, T., \& Gull, T. 2004, ApJ, in press (astro-ph/0404593)

Verner, E., \& Peterson, B. A. 2004, ApJL, in press (astro-ph/0404531) 